\title{
Estudo descritivo da evolução dos gastos pelo Sistema Único de Saúde com internações hospitalares por Acidente Vascular Cerebral em Goiás, 2010-2019
}

\author{
Descriptive study of the evolution of spending by the Sistema Único de Saúde on hospital \\ admissions for stroke in Goiás, 2010-2019
Estudio descriptivo de la evolución del gasto del Sistema Único de Saúde en ingresos hospitalarios por Accidente Cerebrovascular en Goiás, 2010-2019

Paulo Vitor Miranda Macedo de Brito ${ }^{1 *}$, João Vitor Percussor Silva1 ${ }^{1}$, Marcus Vinicius Meneses da Silva1, Laura Abi Faiçal Barros¹, Vinicius da Silva Oliveira' ${ }^{1}$, Arthur Fidelis de Sousa ${ }^{2}$, Giovana de Heberson Souza르. Matheus Henrique Bastos Martins ${ }^{1}$, Antenor Aguiar Almeida Júnior ${ }^{1}$.

\section{RESUMO}

Objetivo: Analisar as os gastos com internação do Sistema Único de Saúde (SUS) com internações por Acidente Vascular Cerebral (AVC) em Goiás, Brasil. Métodos: É um estudo ecológico sobre os gastos públicos com internação por AVC em Goiás, entre janeiro de 2010 e dezembro de 2019. Os dados obtidos por meio do Sistema de Dados do Ministério da Saúde (DATASUS). Foram tabulados pelo programa TabWin® e os calculados pelo Microsoft Exce/®. Não houve submissão ao Comitê de Ética em Pesquisa, por se tratar de um estudo utilizando dados secundários, sem identificação dos participantes. Resultados: Os custos aumentaram em $158,6 \%$ mesmo que as internações abaixaram em $23,8 \%$. Pessoas com mais de 65 anos lideraram a lista de internações com $54,8 \%$. Com relação às tendências, média gasta anual, valor total e intervalo de confiança das dez cidades mais dispendiosas do estado, nenhuma apresentou tendência decrescente. O tratamento de AVC foi responsável por $84,64 \%$ dos procedimentos realizados. Conclusão: As despesas com AVC foram altas e, possivelmente, medidas voltadas à atenção primária diminuiria a despesa total. Além disso, sexo masculino, idade e municípios com maiores portes populacionais e com bons índices de condições de vida são fatores que elevaram as despesas totais.

Palavras-chave: Acidente vascular cerebral, Gastos, Epidemiologia.

\section{ABSTRACT}

Objective: To analyze the costs of hospitalization of the Sistema Único de Saúde (SUS) with hospitalizations for stroke in Goiás, Brazil. Methods: It is an ecological study on public spending on hospitalization for stroke in Goiás, between January 2010 and December 2019. Data obtained through the Sistema de Dados do Ministério da Saúde (DATASUS). They were tabulated by the program TabWin $\circledast$ and those calculated by Microsoft Excel ${ }^{\circledR}$. There was no submission to the Research Ethics Committee, as it is a study using secondary data, without identifying the participants. Results: Costs increased by $158.6 \%$ even though hospitalizations decreased by $23.8 \%$. People over 65 years old led the list of hospitalizations with $54.8 \%$. Regarding trends, average annual spends, total value and confidence interval of the ten most expensive cities in the state, none showed a downward trend. Stroke treatment was responsible for $84.64 \%$ of the procedures performed. Conclusion: Stroke expenses were high and, possibly, measures aimed at primary care would decrease the total expenditure. In addition, male gender, age, and municipalities with larger population sizes and with good levels of living conditions are factors that increased total expenditures.

Keywords: Stroke, Spending, Epidemiology.

1 Universidade Federal de Goiás (UFG), Goiânia - GO. *E-mail: paulovitorgec@gmail.com

${ }^{2}$ Centro Universitário de Anápolis (UniEVANGÉLICA), Anápolis - GO.

SUBMETIDO EM: 10/2020

ACEITO EM: 11/2020

PUBLICADO EM: 1/2021 


\section{RESUMEN}

Objetivo: Analizar los costos de hospitalización del Sistema Único de Saude (SUS) con hospitalizaciones por accidente cerebrovascular en Goiás, Brasil. Métodos: Se trata de un estudio ecológico sobre el gasto público en hospitalización por accidente cerebrovascular en Goiás, entre enero de 2010 y diciembre de 2019. Datos obtenidos a través del Sistema de Dados do Ministério da Saúde (DATASUS). Fueron tabulados por el programa TabWin® y los calculados por Microsoft Excel®. No hubo envío al Comité de Ética en Investigación, por tratarse de un estudio que utiliza datos secundarios, sin identificar a los participantes. Resultados: Los costos aumentaron un $158,6 \%$ a pesar de que las hospitalizaciones disminuyeron un $23,8 \%$. Las personas mayores de 65 años lideraron la lista de hospitalizaciones con un 54,8\%. En cuanto a las tendencias, gasto anual promedio, valor total e intervalo de confianza de las diez ciudades más caras del estado, ninguna mostró una tendencia a la baja. El tratamiento del ictus fue responsable del $84,64 \%$ de los procedimientos realizados. Conclusión: Los gastos por ictus eran elevados y, posiblemente, las medidas dirigidas a la atención primaria disminuirían el gasto total. Además, el sexo masculino, la edad y los municipios con mayor tamaño poblacional y con buenas condiciones de vida son factores que incrementaron el gasto total

Palabras clave: Accidente cerebrovascular, Gasto, Epidemiología.

\section{INTRODUÇÃO}

Acidente vascular cerebral (AVC) é designado como uma insuficiência neurológica aguda causada pela interrupção do fluxo sanguíneo encefálico por oclusão ou ruptura de vasos (BENJAMIN EJ, et al., 2017). Pode ser classificado em dois tipos: AVC hemorrágico e isquêmico.

O primeiro é causado quando um vaso rompe na região intracerebral espontaneamente. O segundo ocorre pela diminuição ou oclusão do fluxo de uma artéria cerebral, levando a isquemia. O tipo isquêmico é responsável por cerca de 85\% dos casos de AVC (BENJAMIN EJ, et al., 2017; BRAININ M, et al., 2018).

Sabe-se que o AVC é a segunda principal causa de morte e incapacidade nos países da América Latina (BENJAMIN EJ, et al., 2017). Entre 1990 e 2017, o número absoluto de pessoas com acidentes vasculares cerebrais aumentou 81\%. Globalmente, o risco de Acidente Vascular Cerebral cresceu de 1990 a 2019 em 9\% (FEIGIN VL, 2019).

Nos Estados Unidos, cerca de 7 milhões de pessoas com idade maior ou igual a 20 anos sofreram pelo menos um episódio de AVC. No Brasil é a principal causa de mortalidade relacionada a causas cardiovasculares, mesmo sabendo-se que os principais Fatores de Risco (FR) para o desenvolvimento da doença são passíveis de acompanhamento e controle na atenção básica (CÂMARA C, 2018; MEDEIROS CS, et al., 2017) .Estudos já mostraram, inclusive, que o principal fator de risco é a Hipertensão Arterial Sistêmica (HAS) (FEIGIN VL, 2019).

Como já sugerido pela Organização Mundial de Saúde (OMS) e demonstrado por diversos estudos, a maneira mais eficaz de reduzir a carga dos acidentes vasculares cerebrais, assim como de outras doenças, é a prevenção primária, isto é, o controle dos FR. No caso do AVC, medidas para a redução do consumo de álcool e tabaco, promoção de alimentação saudável e práticas de exercício são exemplos de estratégias eficientes (MARTINS SC, et al., 2019; FEIGIN VL, 2019).

Contudo, a conscientização pública a respeito do AVC, com a abordagem dos fatores de risco, consequências e reconhecimento de sinais e sintomas ainda é muito precária nos países da América Latina, quando comparados a países de alta renda, ainda que se trate de uma ferramenta simples e pouco custosa (MARTINS SC, et al., 2019).

Em relação às sugestões da OMS, a maioria dos países da América Latina se comprometeu a implementar as estratégias sugeridas. A meta da Ação Global para Prevenção e Controle de Doenças Não Transmissíveis 2013-2020 visa reduzir em $25 \%$ as mortes prematuras por doenças crônicas até 2025 . Em 2018 , foi realizada a 1를 Reunião Ministerial Latino-America de AVC em Gramado, no Brasil, onde foram firmados compromissos para a implementação das medidas cabíveis para a melhor prevenção e tratamento do AVC. Contudo, o progresso observado tem sido lento (MARTINS SC, et al., 2019). 
Desse modo, diante da importância dessa doença no Brasil e no mundo, propõe-se, com este estudo, estimar e analisar o impacto dos gastos públicos com internação do Sistema Único de Saúde (SUS) com internações por Acidente Vascular Cerebral (AVC) no estado de Goiás durante uma década.

\section{MÉTODOS}

Trata-se de um estudo ecológico sobre os gastos públicos com internação por AVC em Goiás, entre o período de janeiro de 2010 e dezembro de 2019. Foram utilizadas informações do Sistema de Dados do Ministério da Saúde (DATASUS), por meio do acesso à informação no TABNET, Assistência à Saúde e do $\mathrm{SIH} / \mathrm{SUS}$.

Os dados coletados referentes à parte financeira, de acordo com o local de residência dos indivíduos, foram o Custo Total das Internações, Valor Total Gasto em Unidade de Terapia Intensiva (UTI) e Custos em Serviços Hospitalares das seguintes doenças: Hemorragia Subaracnoidea (CID10-I60), Hemorragia intracerebral (CID10- 161), outras hemorragias intracranianas não traumáticas (CID10 - I62), Infarto cerebral (CID10-I63), Acidente vascular cerebral, não especificado como hemorrágico ou isquêmico (CID10-I64) e Acidentes vasculares cerebrais isquêmicos transitórios e síndromes correlatas (CID10-G45).

O Custo Total é o valor total relativo às Autorizações de Internações Hospitalares (AlH) aprovadas entre janeiro de 2010 e dezembro de 2019, o Valor dos Serviços Hospitalares é relativo às AlH aprovadas no período e o Valor das Internações em UTI refere-se ao valor total das AlH aprovadas, computadas como internações em UTI, no período estudado. A quantia apresentada não obrigatoriamente reflete o valor repassado aos estabelecimentos. Este valor deve ser considerado como o valor aprovado da produção.

Além disso, foram coletadas informações referentes às internações. Os dados foram separados em procedimentos, faixa etária, diagnósticos, sexo do paciente, caráter da internação e CID. Os procedimentos foram divididos em diagnóstico e/ou atendimento de urgência, tratamento conservador da hemorragia cerebral, tratamento de AVC (Isquêmico ou Hemorrágico Agudo), cirurgia de hematoma intracerebral, procedimentos sequenciais em neurocirurgia. Foram consideradas as faixas etárias: $<15 a, 15-24 a, 25-34 a$, 35-44a, 45-54a, 55-64a e 65 anos e mais. Os diagnósticos foram separados em CID10 G45, 160, 161, I62, 163 e 164, os sexos em masculino e feminino e o caráter das internações em eletivo e urgência.

Também, realizou-se a análise da tendência dos gastos através da regressão linear simples, com obtenção do coeficiente Beta, Intervalo de Confiança e P-Valor, ao nível de significância de $5 \%$. Os dados foram tabulados pelo programa TabWin $\AA$ e os cálculos feitos no programa Microsoft Exce/®. A análise estatística foi realizada no pacote Stata versão 14.0 .

Não houve submissão ao Comitê de Ética em Pesquisa, por se tratar de um estudo utilizando dados secundários, sem identificação dos participantes. A Resolução do Conselho Nacional de Saúde (CNS) nำ466, de 12 de dezembro de 2012, foi atendida, assim como a Resolução de no 510, de 7 de abril de 2016, que dispõe sobre a não necessidade de submissão ao CEP em pesquisas que utilizem informações de domínio público. Também, foram cumpridos os princípios da Declaração de Helsinki.

\section{RESULTADOS E DISCUSSÃO}

Foram analisados 53.042 dados entre o período de 2010 a 2019. A população com mais de 65 anos liderou as internações com $54,9 \%$, seguido por pessoas com idade entre 55 e 64 anos com $20,9 \%$. O procedimento mais descrito foi o Tratamento de AVC (Isquêmico ou Hemorrágico Agudo), com 84,65\% e o tratamento conservador da hemorragia cerebral vem em sequência com $5,53 \%$ das internações.

Os sexos apresentaram diferença de 2,22\% com predomínio da notificação no sexo masculino. No item diagnóstico percebe-se que o CID I64 - Acidente vascular cerebral, não especificado como hemorrágico ou isquêmico - representou $67,34 \%$ das internações, seguido por G45, Acidentes vasculares cerebrais isquêmicos transitórios e síndromes correlata (14,34\%). Esses resultados podem ser vistos na Tabela 1. 
Tabela 1 - Internações divididas em procedimentos, faixa etária, diagnósticos, sexo e caráter e CID em Goiás, entre 2010 e 2019.

\begin{tabular}{|c|c|c|}
\hline Variável & $\mathbf{N}$ & $\%$ \\
\hline \multicolumn{3}{|l|}{ Procedimentos } \\
\hline Diagnóstico e/ou atendimento de urgência & 1993 & 3,75 \\
\hline Tratamento conservador da hemorragia cerebral & 2934 & 5,53 \\
\hline Tratamento de AVC (Isquêmico ou Hemorrágico Agudo) & 44900 & 84,64 \\
\hline Cirurgia de Hematoma Intracerebral & 539 & 1,01 \\
\hline Procedimentos Sequenciais em neurocirurgia & 503 & 0,95 \\
\hline Outros & 2.173 & 04 \\
\hline \multicolumn{3}{|l|}{ Faixa Etária } \\
\hline$<15$ & 285 & 0,51 \\
\hline $15-24 a$ & 743 & 1,4 \\
\hline $25-34 a$ & 1519 & 2,7 \\
\hline $35-44 a$ & 3442 & 6,3 \\
\hline $45-54 a$ & 7031 & 13,2 \\
\hline 55-64a & 11128 & 20,9 \\
\hline $65 e+a$ & 28894 & 54,9 \\
\hline Total & 53042 & 100 \\
\hline \multicolumn{3}{|l|}{ Diagnóstico } \\
\hline G45 & 7658 & 14,34 \\
\hline 160 & 2676 & 5,04 \\
\hline 161 & 4875 & 9,19 \\
\hline 162 & 775 & 1,46 \\
\hline $163-$ & 1335 & 2,51 \\
\hline 164 & 35723 & 67,34 \\
\hline Total & 53042 & 100 \\
\hline \multicolumn{3}{|l|}{ Sexo } \\
\hline Masculino & 28083 & 51,11 \\
\hline Feminino & 24959 & 48,89 \\
\hline Total & 53042 & 100 \\
\hline \multicolumn{3}{|l|}{ Caráter da Internação } \\
\hline Eletivo & 776 & 1,45 \\
\hline Urgência & 52266 & 98,5 \\
\hline Total & 53042 & 100 \\
\hline
\end{tabular}

Fonte: Brito PV, et. al, 2020; dados extraídos do Departamento de Informática do SUS-DATASUS.

Com relação às tendências, média gasta anual, valor total e intervalo de confiança relacionadas ao Custo Total, Custo das Internações em UTI e Custo dos Serviços Hospitalares, nas dez cidades goianas mais dispendiosas, estão dispostas na Tabela 2.

Nenhuma cidade apresenta tendência decrescente. Além disso, o Custo Total das seguintes cidades mostram tendências crescentes: Goiânia, com média anual de gasto de $R \$ 2.531 .796,417$ ( $\beta=54834.23 \mathrm{e}$ IC $=133749.3$ a 212664.3), Aparecida de Goiânia, com $R \$ 978.674$ ( $\beta=59260.85$ IC =25603.25 a 92918.44), Itumbiara, com $R \$ 155.066$ ( $\beta=15743.56$ e IC $=5.184 .279$ a 26302.84 e Caldas Novas, com $R \$ 117.751$ ( $\beta$ $=12112.67$ e IC $=6.984 .102$ a 17241.23$)$. 
Tabela 2 - Valor acumulado, coeficiente Beta, Intervalo de Confiança, P-valor e tendência do Custo Total, Custo da Internação em UTI e Custo Serviços Hospitalares com CID G45, I60-165 em Goiás, entre 2010 e 2019, por cidade.

\begin{tabular}{|c|c|c|c|c|c|c|}
\hline Cidade & $\begin{array}{l}\text { Valor } \\
\text { Acumulado }\end{array}$ & Beta & \multicolumn{2}{|c|}{ Intervalo de confiança } & P-Valor & Tendência \\
\hline \multicolumn{7}{|l|}{ Custo Total } \\
\hline Anápolis & $\mathrm{R} \$ 7.120 .509$ & 15613.86 & -16173.01 & 47400.72 & 0.290 & Estacionário \\
\hline $\begin{array}{l}\text { Aparecida de } \\
\text { Goiânia }\end{array}$ & $\mathrm{R} \$ 9.786 .747$ & 59260.85 & 25603.25 & 92918.44 & 0.004 & Crescente \\
\hline Caldas Novas & $\mathrm{R} \$ 1.177 .512$ & 12112.67 & 6.984 .102 & 17241.23 & 0.001 & Crescente \\
\hline Catalão & $\mathrm{R} \$ 1.336 .956$ & -3.065 .279 & -15930.33 & 9799.769 & 0.598 & Estacionário \\
\hline Goiânia & $\mathrm{R} \$ 25.317 .964$ & 133749.3 & 54834.23 & 212664.3 & 0.004 & Crescente \\
\hline Itumbiara & $\mathrm{R} \$ 1.550 .664$ & 15743.56 & 5184.279 & 26302.84 & 0.009 & Crescente \\
\hline Jataí & $\mathrm{R} \$ 1.389 .788$ & 5.716 .201 & -7238.662 & 18671.06 & 0.339 & Estacionário \\
\hline Rio Verde & $\mathrm{R} \$ 1.817 .138$ & 9.655 .937 & -4389.776 & 23701.65 & 0.152 & Estacionário \\
\hline Senador Canedo & $\mathrm{R} \$ 2.069 .278$ & 6.741 .938 & -11117.76 & 24601.64 & 0.409 & Estacionário \\
\hline Trindade & $\mathrm{R} \$ 2.558 .259$ & 10280.22 & -8.342 .891 & 28903.33 & 0.239 & Estacionário \\
\hline Estado & $\mathrm{R} \$ \mathbf{8 9 . 0 9 7 . 2 4 9 , 5 2}$ & 580067.1 & 485661.6 & 674472.5 & 0 & Crescente \\
\hline \multicolumn{7}{|c|}{ Custo internação em UTI } \\
\hline Anápolis & $\mathrm{R} \$ 3.482 .209$ & 17524.05 & -5543.488 & 40591.59 & 0.118 & Estacionário \\
\hline $\begin{array}{l}\text { Aparecida de } \\
\text { Goiânia }\end{array}$ & $\mathrm{R} \$ 5.104 .112$ & 29406.83 & 4662.97 & 54150.68 & 0.025 & Crescente \\
\hline Caldas Novas & $\mathrm{R} \$ 687.513$ & 9.936 .921 & 4737.086 & 15136.76 & 0.002 & Crescente \\
\hline Catalão & $\mathrm{R} \$ 777.949$ & -2.876 .491 & -12952.54 & 7.199 .553 & 0.529 & Estacionário \\
\hline Goiânia & $R \$ 13.589 .624$ & 71803.99 & -9179.258 & 152787.2 & 0.075 & Estacionário \\
\hline Itumbiara & $\mathrm{R} \$ 673.912$ & 9.322 .389 & -1029.455 & 19674.23 & 0.071 & Estacionário \\
\hline Jataí & $\mathrm{R} \$ 693.665$ & 2.979 .669 & -7276.523 & 13235.86 & 0.522 & Estacionário \\
\hline Rio Verde & $\mathrm{R} \$ 877.493$ & 3.234 .986 & -4839.196 & 11309.17 & 0.383 & Estacionário \\
\hline Senador Canedo & $\mathrm{R} \$ 1.559 .285$ & 7.299 .554 & -10322.39 & 11782.3 & 0.883 & Estacionário \\
\hline Trindade & $\mathrm{R} \$ 1.049 .341$ & 4.916 .269 & -7926.615 & 17759.15 & 0.403 & Estacionário \\
\hline Estado & $R \$ 46.045 .237$ & 324320.9 & 213669 & 434972.9 & 0 & Crescente \\
\hline \multicolumn{7}{|c|}{ Custo Serviços Hospitalares } \\
\hline Anápolis & $\mathrm{R} \$ 6.074 .687$ & 12642.07 & -14578.29 & 39862.43 & 0.315 & Estacionário \\
\hline $\begin{array}{l}\text { Aparecida de } \\
\text { Goiânia }\end{array}$ & $\mathrm{R} \$ 8.347 .074$ & 50386.75 & 22206.41 & 78567.09 & 0.003 & Crescente \\
\hline Caldas Novas & $\mathrm{R} \$ 1.012 .069$ & 10568.11 & 6152.72 & 14983.5 & 0.001 & Crescente \\
\hline Catalão & $\mathrm{R} \$ 1.125 .961$ & -2860.06 & -13783.48 & 8.063 .363 & 0.563 & Estacionário \\
\hline Goiânia & $\mathrm{R} \$ 21.796 .538$ & 120053.6 & 54197.73 & 185909.6 & 0.003 & Crescente \\
\hline Itumbiara & $\mathrm{R} \$ 1.324 .360$ & 13355.74 & 4567.826 & 22143.65 & 0.008 & Crescente \\
\hline Jataí & $\mathrm{R} \$ 1.191 .670$ & 4.709 .828 & -6377.161 & 15796.82 & 0.356 & Estacionário \\
\hline Rio Verde & $\mathrm{R} \$ 1.554 .564$ & 8.700 .847 & -3270.837 & 20672.53 & 0.132 & Estacionário \\
\hline Senador Canedo & $\mathrm{R} \$ 2.183 .147$ & 5.534 .042 & -9270.835 & 20338.92 & 0.414 & Estacionário \\
\hline Trindade & $\mathrm{R} \$ 1.774 .261$ & 8.914 .138 & -6931.387 & 24759.66 & 0.231 & Estacionário \\
\hline Estado & $\mathbf{R} \$ 76.308 .838$ & 503773.4 & 425518.2 & 582028.6 & 0 & Crescente \\
\hline
\end{tabular}

Fonte: Brito PV, et. al, 2020; dados extraídos do Departamento de Informática do SUS-DATASUS. 
Ao observar o custo da internação em UTI percebe-se que há tendências crescentes apenas nas cidades de Aparecida de Goiânia $(\beta=29406.83 \mathrm{IC}=4662.97$ a 54150.68) e Caldas Novas $(\beta=9.936 .921$ e IC= 4.737.086 a 15136.76) e a média anual de cada uma foi de $R \$ 510.411$ e $R \$ 68.751$, respectivamente. Em relação, ao Custo dos Serviços Hospitalares, Aparecida de Goiânia $(\beta 50386.75 \mathrm{IC}=22206.41$ a 78567.09$)$ e Caldas Novas ( $\beta=10568.11$ e IC $=6152.72$ a 14983.5) possuem tendências crescentes e $R \$ 834.707$ e $R \$$ 101.206 de média anual de gasto respectivamente. Por fim, o custo total com os CIDs G45, I60-165 em Goiás foi de $R \$ 89.097 .249$, o custo de internação por UTI foi $R \$ 46.045 .237$ e custo dos serviços hospitalares $R \$$ 76.308.838, as três medidas apresentam tendência crescente no estado.

No estado de Goiás, nos anos de 2010 a 2019, observou-se 53.042 internações por AVC, sendo gastos um total de $\mathrm{R} \$ 89.097 .249$, com tendência temporal crescente, sendo o município de Goiânia responsável por $28,4 \%$ dos gastos.

O sexo masculino teve o maior número de internações, $51,11 \%$, o que pode se associar com a maior prevalência dos principais fatores de risco nessa população, como o alcoolismo e o tabagismo (O'DONNELL, et al., 2010). Em um estudo que avaliou a prevalência e a incapacidade associada ao AVC encontrou prevalência pontual de 1,6\% em homens e 1,4\% em mulheres, e de incapacidade $29,5 \%$ em homens e de $21,5 \%$ em mulheres (BENJAMIN EJ, et al., 2017).

Outro estudo demonstrou que os anos de vida perdidos ajustados por incapacidade (DALY) são mais significativos em homens de 50 a 69 anos em que ainda são economicamente ativos. O que está associado a maiores custos da doença para os cofres públicos, tanto de forma direta, com diárias hospitalares, exames complementares, medicamentos e honorários médicos, como com custos indiretos, como perda de produtividade associada ao absenteísmo ou à mortalidade precoce (BENJAMIN EJ, et al 2017; CÂMARA C, 2018).

O maior número de internações se encontra na população com 65 anos ou mais, cerca de $75,9 \%$. É fato que a idade é um fator de risco independente para o AVC. A maior parte dos eventos de AVC ocorrem a partir dos 65 anos de idade, dobrando o risco a cada década após essa idade (BOTELHO, et al., 2016). A magnitude desse problema em idosos é expressiva, no Brasil as Doenças cerebrovasculares são a $4^{\circ}$ causas de internações entre idosos, atrás de insuficiência cardíaca, bronquite/enfisema e outras doenças pulmonares obstrutivas crônicas e pneumonia (VIEIRA LG, et al., 2019).

Cerca de $50 \%$ dos pacientes que sobrevivem ao um episódio de AVC apresenta incapacidades permanentes e significantes, que requerem assistência e supervisão (MARQUES S, 2006). Problemas de saúde geral, problemas psiquiátricos, incontinência urinária, dor, comprometimento motor e histórico de quedas recorrentes são consequências do AVC na população idosa.O que de uma maneira indireta gera gastos imensuráveis para o sistema de saúde pública e compromete a qualidade de vida da população idosa e de seus cuidadores (LUI SK, 2018).

O AVC não especificado foi o mais cadastrado como causa de internação $(67,34 \%)$, já o acidente vascular cerebral isquêmico (AVCl) correspondeu a apenas 2,51\% do total do número de internações e o acidente vascular cerebral hemorrágico (AVCH) correspondeu a 9,19\%. Isso denota falhas no preenchimento dos dados que são enviados para o $\mathrm{SIH}$, uma vez que a literatura traz o $\mathrm{AVCl}$ como mais prevalente, podendo chegar a cerca de $88 \%$ dos casos (BARROS AC, et al., 2009).

A falta de treinamento dos profissionais para preencherem as autorizações de internações hospitalares e a própria ausência de elucidação diagnóstica devido à falta de capacitação ou exames (como tomografia) pode explicar essas divergências de dados. O que pode diminuir a confiabilidade dos dados do SIH/SUS, bem como limitar a possibilidade de utilizar esses dados para analisar o perfil e monitorizar o AVC em Goiás. Isso dificulta o planejamento de políticas públicas que visem reduzir os números de internações por AVC em Goiás, uma vez que dados válidos e confiáveis são fundamentais para tomada de decisões e a programação das ações de saúde (CASTRO CD, 2013, BATISTA SRR, et al., 2012).

Os gastos com Doenças cerebrovasculares são dispendiosos e consomem boa parte da verba pública. Segundo o DataSUS, entre 2010 e 2019, o Acidente Vascular Cerebral representou 1,2\% das internações e $1,4 \%$ do valor hospitalar total gasto em Goiás, considerando todas as causas. Em um estudo realizado em 
2017, foi observado a evolução dos gastos com internações hospitalares por condições sensíveis à atenção primária (ICSAP) no Brasil, de 2000 a 2013, e os dados evidenciaram que as doenças do aparelho circulatório, em especial insuficiência cardíaca, angina e doenças cerebrovasculares, contribuíram para a maior parte dos gastos (SOUZA DK, et al., 2009).

Um estudo que avaliou os custos por AVC em um hospital público de Joinville, Santa Catarina, entre os anos de 2016 e 2017, encontraram um custo total de US \$1,1 milhão (VIEIRA LG, et al., 2019). Esses dados mostram que a doença em questão representa um percentual expressivo dos gastos do serviço público. Os números só tendem a se elevar com o decorrer dos anos se for considerado apenas fatores pontuais, como o envelhecimento da população.

O país encontra-se em transição demográfica, em rápido processo de envelhecimento e o Índice de Envelhecimento populacional está em processo de elevação. Estudos identificaram que na região centrooeste, em onze anos (2000-2010), houve um aumento 3\% na população com mais de 60 anos e uma elevação do Índice de envelhecimento de $22,1 \%$ para $36,0 \%$. Assim, pode-se notar uma das razões pelas quais a população acima de 55 anos em Goiás foi responsável por cerca de $74 \%$ das internações.

Nos Estados Unidos foi realizado um estudo para avaliar as tendências de internação por AVC no National Hospital Discharge Survey entre o ano de 1988 a 1997. No geral, 58\% dos AVCs foram classificados como isquêmicos, $13 \%$ como hemorrágicos e $29 \%$ não especificados (FANG J, MICHAEL HA., 2001).

$\mathrm{Na}$ Alemanha houve um estudo com resultado parecido, em uma pesquisa com 242.090 pessoas com intuito de avaliar a prevalência dos CIDS I60-I64 observou-se que de 1.263 casos apenas 3,32\% não foi classificado como isquêmico ou hemorrágico (EGEN-LAPPE, et al., 2013).

Diferentemente dos resultados obtidos nesses estudos, o Centro-Oeste brasileiro segue uma tendência de não especificidade do diagnóstico. Segundo dados do DataSUS, ao analisar apenas os CIDS IG45 e I64 na região, durante os anos de 2010-2019, observa-se que 92\% dos diagnósticos não há diferenciação entre AVC isquêmico ou hemorrágico e em Goiás esse valor foi $78.7 \%$. Dessa forma, é nítido a diferença entre a especificidade do diagnóstico de AVC em países desenvolvidos e o Brasil e a não diferenciação entre AVC isquêmico e hemorrágico provavelmente afeta o prognóstico, o tempo de tratamento e a despesa públicas com a doença.

Um dos fatores relacionados à diferença entre a especificidade dos diagnósticos de Goiás e dos países analisados no parágrafo anterior, pode estar relacionado ao investimento na área da saúde. Por exemplo, em 2018 os Estados Unidos ultrapassaram US $\$ 10$ mil per capita em investimento na saúde, a Alemanha investiu US\$ 5.986, já o Brasil investiu apenas US\$ 1.282 per capita (OECD, 2019). Com mais investimento, provavelmente, há mais aparelhos necessários para a realização do diagnóstico e, consequentemente, há menos dúvida entre os CIDs.

Mais de $98 \%$ dos pacientes foram internados por urgência e mais de $80 \%$ dos procedimentos foram realizados para o tratamento da doença (DATASUS, 2020). O tratamento do AVC é dispendioso e quanto mais procedimentos necessários para o tratamento mais se torna caro, um estudo realizado em hospitais privados de Joinville mostrou isso. O custo mediano por paciente de acordo com o diagnóstico das internações foram os seguintes: US\$ $3.827 \mathrm{com}$ AVC isquêmico, US\$2.315 com hemorragia subaracnoidea/ataque isquêmico transitório, US\$ $16.442 \mathrm{com}$ hemorragia intracerebral primária e US\$28.928 com hemorragia subaracnoidea. Pacientes submetidos a trombectomia intra-arterial, o custo foi de US\$35.092. Portanto, é nítido que por se tratar de uma doença passível de prevenção primária o custo é relativamente alto (VIEIRA LG, et al. 2019; MARTINS SC, et al., 2019).

Apenas entre 2012 e 2013, o custo médico direto com AVC estimado globalmente foi de 17,9 bilhões de dólares (BENJAMIN EJ, et al., 2017). Apesar da carência de dados acerca dos custos com AVC, especialmente nos países de baixa renda, estima-se que o custo imposto por tal patologia seja responsável por 2 a $4 \%$ de todos os gastos com saúde no mundo. Os Estados Unidos, país com os maiores gastos em saúde no mundo, teve um gasto aproximado de US\$18,8 bilhões. A China, país com a maior incidência de AVC globalmente, teve um custo anual de aproximadamente US\$6 bilhões (ZHANG H, et al., 2019). Em 
relação ao Brasil, no ano de 2019, pelo DATASUS, os Acidentes Vasculares Cerebrais (AVC) responderam por um custo total superior a 253 milhões de reais. Isto representa 1,6\% do valor total gasto com internações hospitalares no mesmo ano, com aumento de $73,8 \%$ das despesas de internação com AVC quando comparado com 2010.

Durante o período contemplado no presente estudo (2010-2019), a região com maior número absoluto de internações, segundo dados obtidos pelo DATASUS, foi a Sudeste, com São Paulo liderando tal estatística. O local com menos internações foi a Região Norte. Já a região Centro-Oeste ficou em 4 ㅇ lugar no número de internações, com o estado de Goiás em primeiro lugar. Observando a tendência ao longo dos anos do estudo, ainda que a Região Centro-Oeste tenha se mantido constante em sua posição no ranking, os números absolutos e relativos tanto com as internações por AVC como com seus gastos se mantiveram em ascensão. Isso corrobora mais uma vez para a relevância do presente estudo.

Em Goiás, comparativamente com o Brasil como um todo os números são ainda mais expressivos, com aumento de custos de $158,6 \%$ apenas nessa modalidade de internação, ainda que o aumento no número de internações tenha sido muito inferior: $23,8 \%$. Ao mesmo tempo em que o envelhecimento e o surgimento de novas tecnologias possam contribuir para o aumento de custos, não se pode ignorar a existência de outras possíveis explicações para este cenário econômico. Ainda que o aumento da prevalência de AVC seja uma realidade documentada (LINDSAY MP et al., 2019), a incidência em muito poderia ser diminuída caso medidas de prevenção primária fossem instituídas.

Como já exposto pela própria OMS e diversos estudos, esta é a medida mais eficaz tanto para o freio no número de casos como para, consequentemente, a diminuição custos (BRAININ M, 2018; STEIGER NC, 2016; MARTINS SC, 2019).

Não obstante, a escassez na padronização propedêutica do AVC pode levar ao uso indiscriminado e pouco eficiente de ferramentas diagnósticas e terapêuticas custosas, sem que isso traga retorno ao paciente. A maior parte dos municípios que tiverem tendências crescentes do gasto com AVC estão na região metropolitana de Goiânia, segundo a "lei dos cuidados inversos" os municípios com maior porte populacional, pertencentes à região metropolitana, próximos à capital e com melhores índices de condições de vida e saúde tem maior tendência de apresentarem maiores taxas de internação e por consequência maiores gastos (VIEIRA LG, et al., 2019).

Por outro lado, Senador Canedo e Anápolis tiveram tendências estacionárias de gastos, o que poderia sugerir uma maior dificuldade de acesso aos serviços de saúde ou ainda uma evasão dos pacientes para hospitais pertencentes ao município de Goiânia. Ainda são raros estudos que analisem a tendência de gastos com AVC no Brasil. Estudo que analisou os gastos com AVC na Inglaterra em 2015 e fez projeções para gastos em 2035 concluíram que haverá um aumento de 194\% em 20 anos nos gastos por AVC, indo de £26 bilhões em 2015 a $£ 75$ bilhões em 2035 (CASTRO CD, 2013).

As limitações do presente estudo são em relação à fonte de dados $(\mathrm{SIH})$, os valores podem estar subestimados uma vez que esses sistemas não estão totalmente sedimentados e consideram somente dados que se referem às internações reembolsadas pelas SUS. Sabe-se que cerca de $24,2 \%$ da população brasileira possuem plano privado de saúde, sendo $26,9 \%$ entre os idosos, e, portanto, não foram contabilizados nos custos de internações hospitalares Além de não levar em conta outras fontes de financiamento no orçamento dos hospitais (PEIXOTO SV, et al., 2004).

\section{CONCLUSÃO}

Os resultados encontrados neste estudo sugerem que as despesas com AVC em Goiás são altas e consomem boa parte da verba pública, além disso, o gasto teve uma tendência crescente ao longo do período analisado. Um dos possíveis motivos para tal é a falha de medidas voltadas à atenção básica, pois o risco de AVC sabidamente pode ser reduzido em até $90 \%$ com ações voltas à atenção primordial e primaria. Outro fator que pode ter elevado os gastos foi o alto número de diagnósticos $(70.7 \%)$ não diferenciados entre AVC entre isquêmico e hemorrágico, isso afeta o afeta o prognóstico, o tempo de tratamento e, possivelmente, a despesa pública com a doença. O sexo masculino teve o maior número de internações, o que pode se associar com a maior prevalência alcoolismo e o tabagismo nessa população, que são fatores de risco para 
o AVC. A idade mais afetada foi a maior que 65 anos, pois o envelhecimento é um fator independente para o desenvolvimento de AVC.

\section{REFERÊNCIAS}

1. BATISTA SRR, et al. Hospitalizações por condições cardiovasculares sensíveis à atenção primária em municípios goianos. Rev. Saúde Pública, 2012; 46(1):34-42.

2. BARROS AC, et al. Abordagem inicial e conduta no acidente vascular encefálico isquêmico agudo. Rev. Méd. Minas Gerais, 2009; 19(4, supl.3):S48-52.

3. BENJAMIN EJ, et al. Heart disease and stroke statistics-2017 update. American Heart Association, 2017; 136(3), e1e23.

4. BOTELHO TS. Epidemiologia do acidente vascular cerebral no Brasil. Revista Brasileira de. Epidemiologia, 2016; 19(1):122.

5. BRAININ M, et al. Cut stroke in half: polypill for primary prevention in stroke. Int J Stroke, 2018; 13: 633-47.

6. CÂMARA C. Mapeamento político da saúde no Brasil com foco em diabetes e doenças cardiovasculares. Mapeamento político da saúde no Brasil com foco em diabetes e doenças cardiovasculares, 2018; 210-210p.

7. CASTRO CD. Estudo de confiabilidade dos dados do Sistema de Informações Hospitalares. Brasil, 2013;1-125.

8. EGEN-LAPPE, et al. Incidence estimate and guideline-oriented treatment for post-stroke spasticity: an analysis based on German statutory health insurance data. International journal of general medicine, 2013; 135p.

9. FANG J, MICHAEL HA. Trend of stroke hospitalization, United States, 1988-1997. STROKE-DALLAS, 2001; 22212225.

10. FEIGIN VL. Anthology of stroke epidemiology in the 20th and 21st centuries: Assessing the past, the present, and envisioning the future. International Journal of Stroke, 2019; 223-237p.

11. LIMA CM, et al. Características epidemiológicas e clínicas dos pacientes acometidos por acidente vascular cerebral. J Health Sci Inst, 2015; 33(1), 45-49.

12. LINDSAY MP, et al. "World Stroke Organization (WSO): global stroke fact sheet, 2019; 806-817.

13. MARTINS SC. Ouriques et al. Priorities to reduce the burden of stroke in Latin American countries. The Lancet Neurology, 2019; 18(7), 674-683.

14. MEDEIROS CS, et al. Perfil social e funcional dos usuários da Estratégia Saúde da Família com Acidente Vascular Encefálico. Rev. Bras. Cienc. Saud. 2017; 21(3), 211-20.

15. MINISTÉRIO DA SAÚDE - Sistema de Informações Hospitalares do SUS (SIH/SUS).

16. O'DONNELL MJ, et al. Risk factors for ischaemic and intracerebral haemorrhagic stroke in 22 countries (the INTERSTROKE study): a case-control study. The Lancet, 2010; 376(9735), 112-123.

17. OECD. Organization for Economic Co-operation and Development. Health at glance: OECD indicators. Paris, France 2019; 320p.

18. PEIXOTO SV, et al. Custo das internações hospitalares entre idosos brasileiros no âmbito do Sistema Único de Saúde. Epidemiologia e Serviços de Saúde, 2004; 13(4), 239-246.

19. SOUZA DK et al. Estudo descritivo da evolução dos gastos com internações hospitalares por condições sensíveis à atenção primária no Brasil, 2000-2013. Epidemiol e Serviços Saúde, 2017; 26(2):285-94.

20. STEIGER NC. Primary prevention of stroke. Jama, 2016; 316: 658-59.

21. VIEIRA LG, et al. O custo do AVC em hospitais privados no Brasil: um estudo prospectivo. Arquivos de NeuroPsiquiatria, 2019; 77(6), 404-411.

22. ZHANG H, et al. Costs of hospitalization for stroke from two urban health insurance claims data in Guangzhou City, southern China. BMC health services research, 2019; 19(1), 671. 